\title{
Piterari/der Anzeiger
}

\section{ju Den \\ (Entideidungen bes Reidşgeridt?.}

Berlag von Beit \& Comp. in Reipzig.

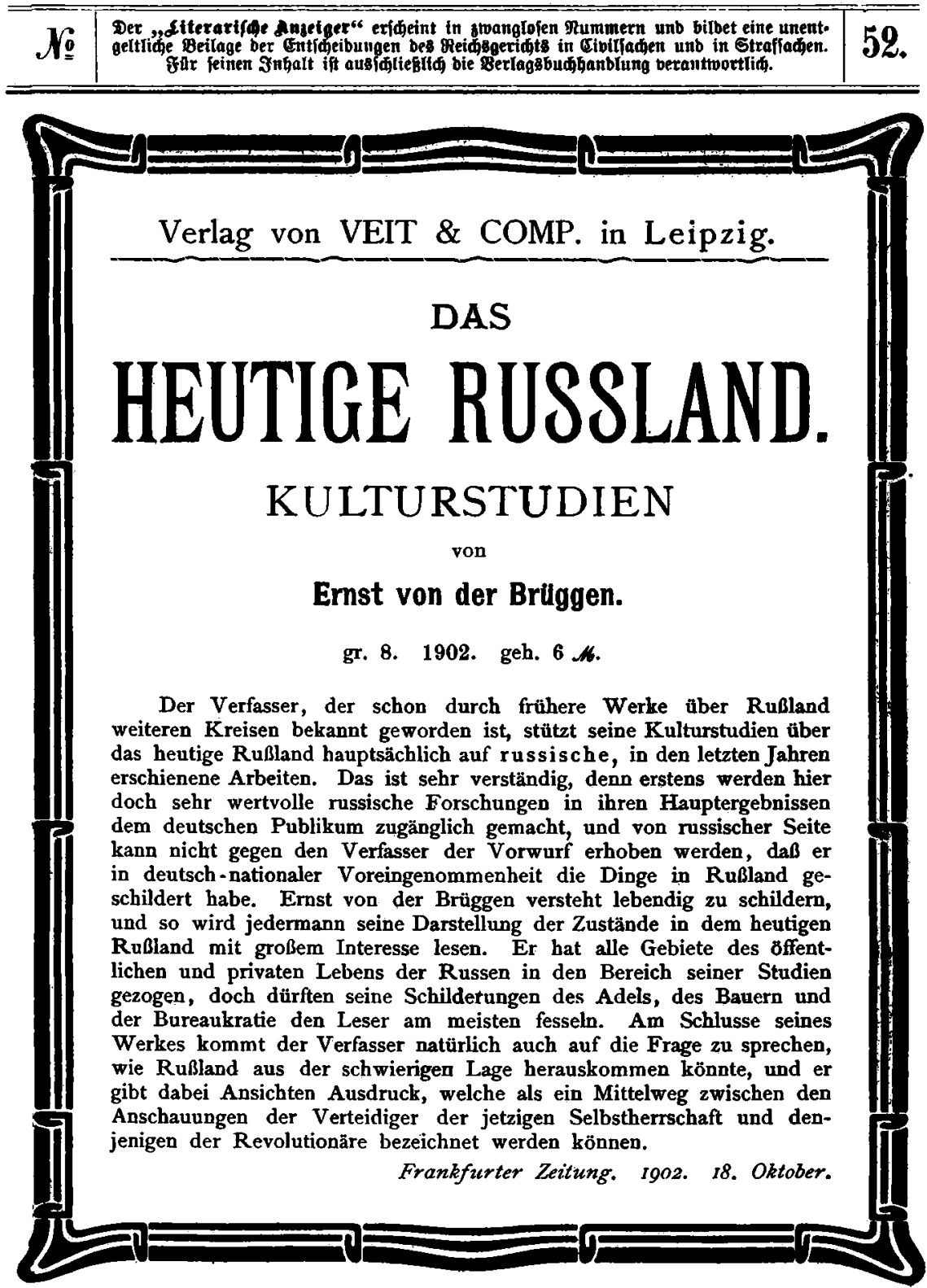




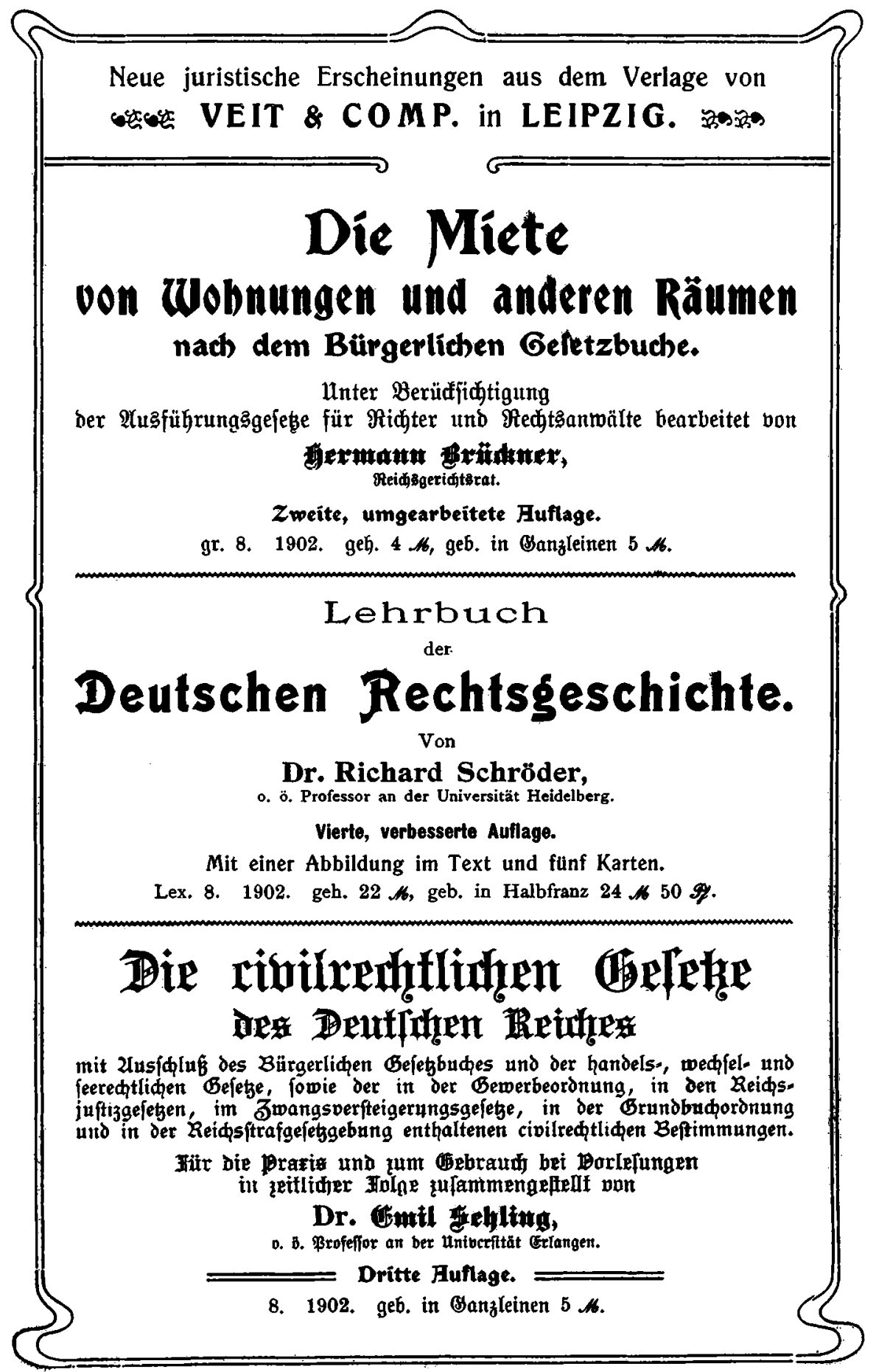




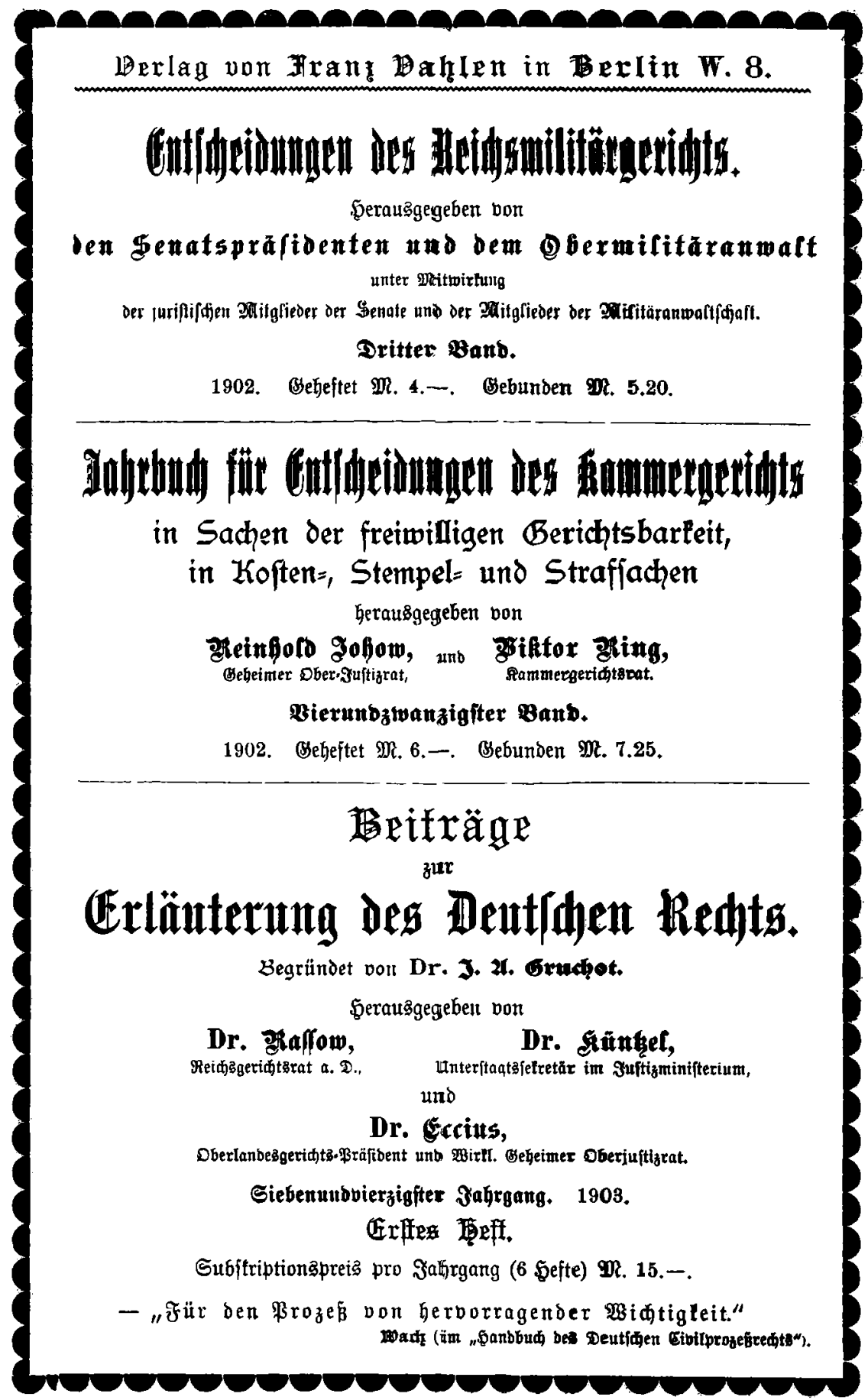




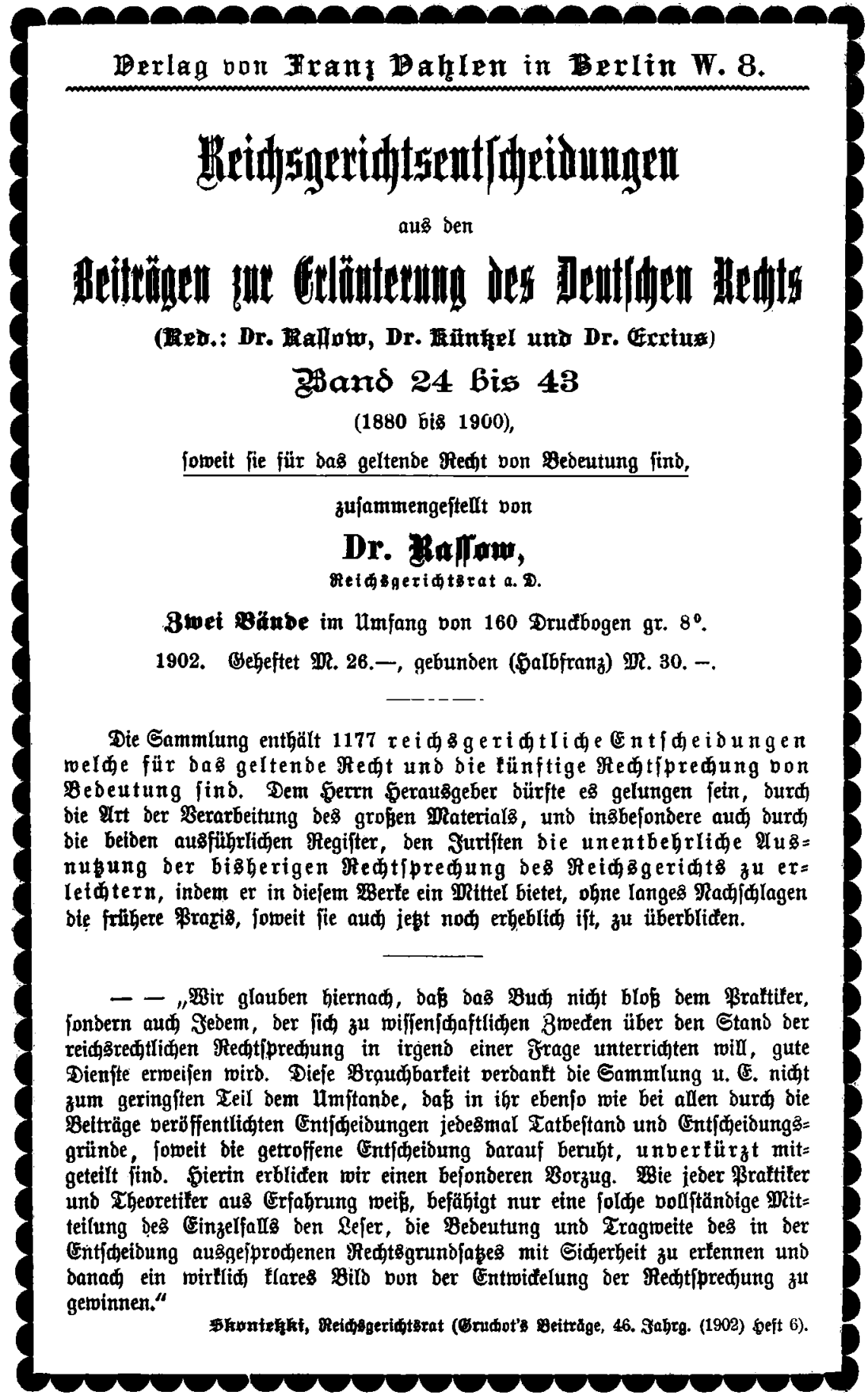




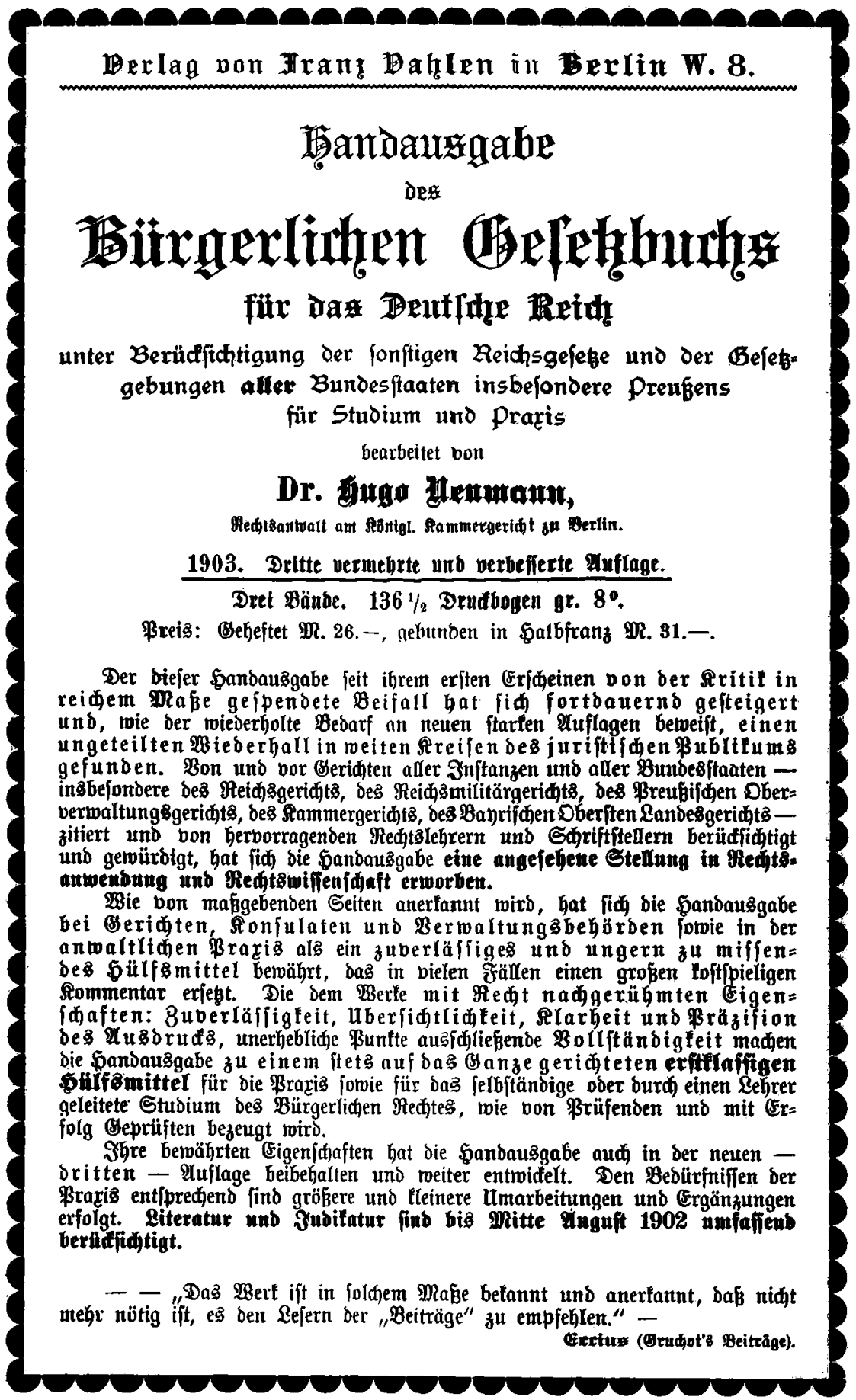




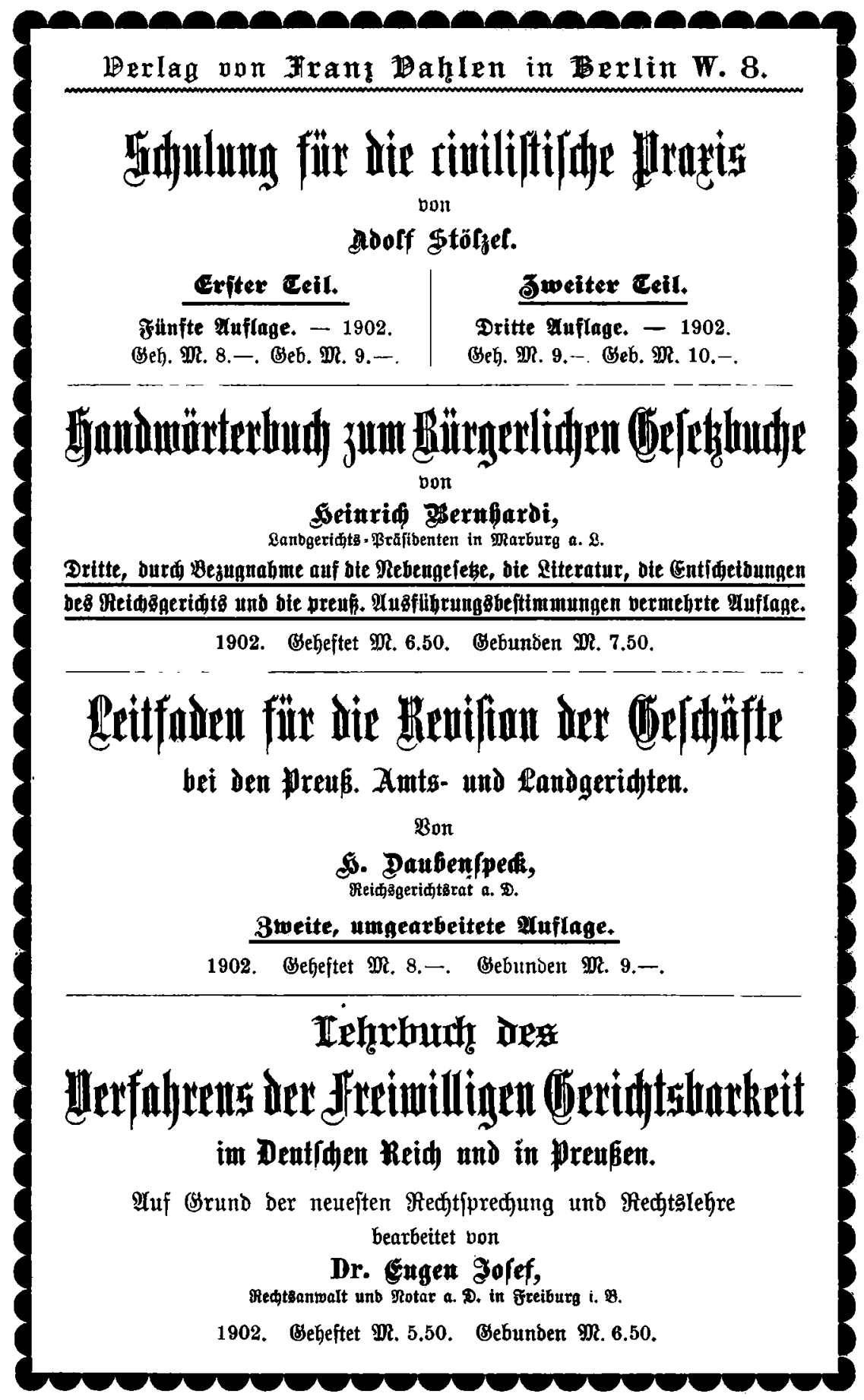




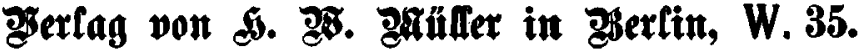

Soeben erifienten:

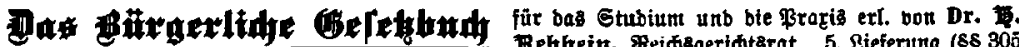
biš 386 umfaffent). MR. 3.50 .

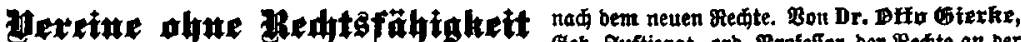

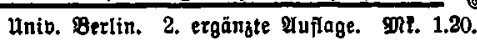

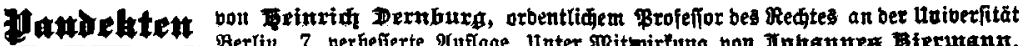
Berlin. 7. verbefferte Iuflage. Inter Mitwirtung bon Iv hamute Birrutann,

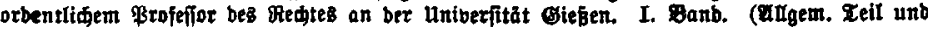
Sadentedt.) MRe. 14.-; geb. MRt. 15.75.

Banb II unb III eridjeinen in einigen Stonaten.

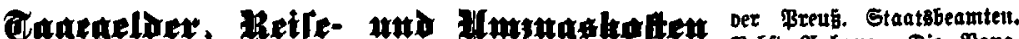
Rebít Mrhang: Die Mang.

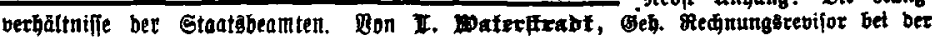
Dbet.Redhungżtammer. Mt. 7. -; geb. פRt. 8.60.

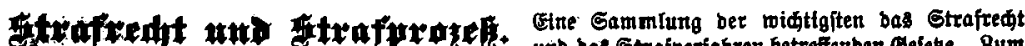

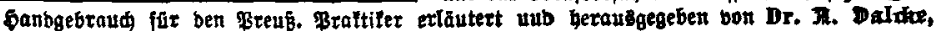

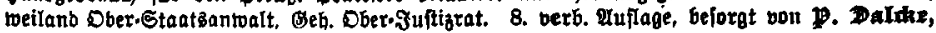
antertidter. (Gé). Dit. 8.50.

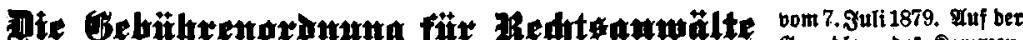
(B) tars bon anwalt beim Rammergerifft. 4. Aufl. 1. u. 2. Mbteilung a git. 3.-.

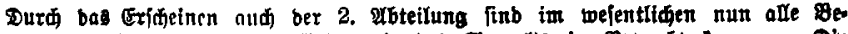

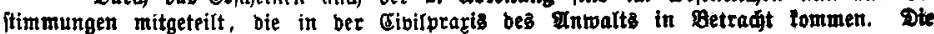

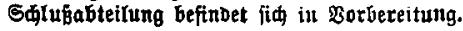

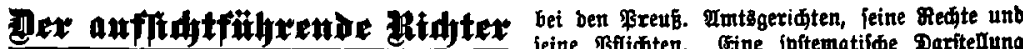

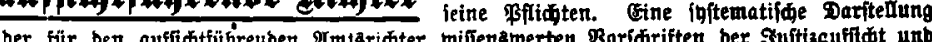

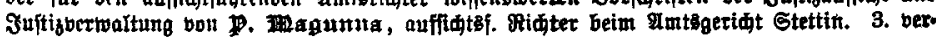

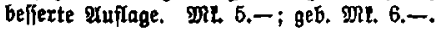

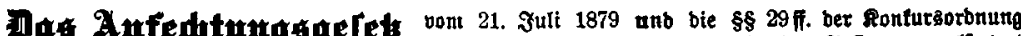
Anfedytumg\$geres in ben feit 1 . Sanuar 1900 geltenben Fafiungen, etlāutert burd bie Entideibungen DeE Reidggerifts bon 1 . IukN, Juftigrat. 2. vermehrte थ̈ufluge. IRt. 1.20 .

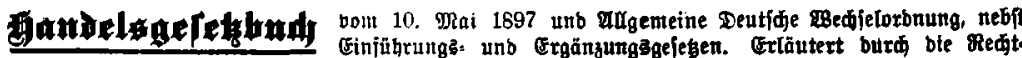

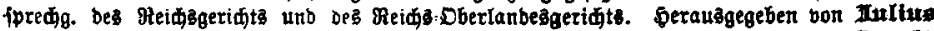

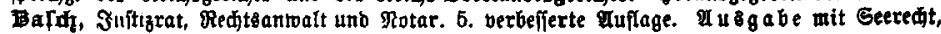
geb. Mit. 4.一.

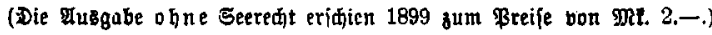

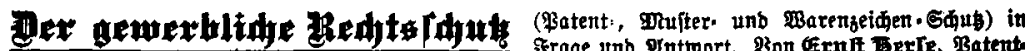
antoalt. I. Teil: Deutifes Stcid. Sartoniert Mit. 4.-

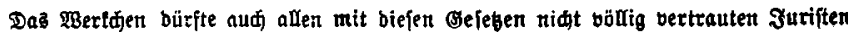
zur Drientierung willommen feir. 


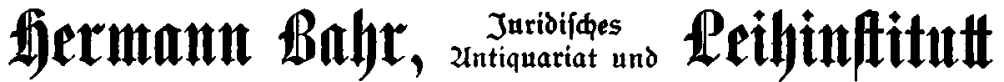 rechts: und praatswiffenichaftlicher citeratur. \\ ca. 300,000 bände.}

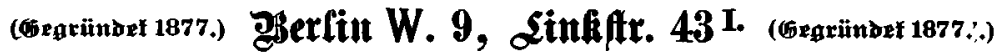
Webingungen des Eeibininfituts auf Deriangen.

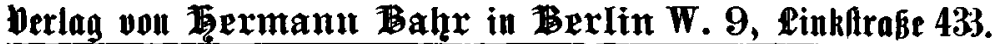

\section{Das Deutide Medielredpt}

mit erläuterndell formularen

für den afademifhen Gebraud bearbeitet pon

c. zxep, Doftor der Rechte.

3. verbel|rett und vermehrte Xuflage

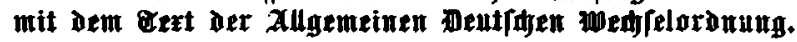

1901. XIII, 339 S. $8^{\circ}$. Preis brofichert $4.50 \mathrm{~m}$., elegant gebunden $5 \mathrm{~mm}$.

\section{Das Hixdyented}

mit Cin/doluß deg Cljeredhts in frage und Autwort

bearbeitet von Dr. jur. C. HeY.

1895. $18 \frac{1}{2}$ Drudbogen. Ungebunden $4 \mathfrak{m}$., in eleg. Eeinenband $4.60 \mathrm{~mm}$.

Er Ju bejiegen ourd alle Buchgandingen.

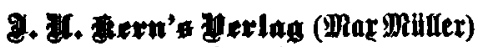
in Prealau.

Soeben volftändig gemorben:

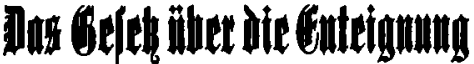 woun Gentudetinethithun}

bom 11. Suni 1874.

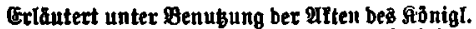
\$reur. Minifteriums ber bffeutf́tgen arbeiten bou Dr. jur. Georg Eger, Reg.sat.

Brweite bermefrtte $\mathfrak{A}$ uffage 1902. critex Baub.

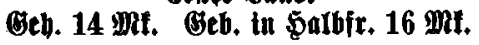
Bweiter gand.

Geth. 15 Mf. Geb, in barbir. 17 Mt.

„Der Eger'jife \$ommentar bilbet ble be. beutenofte uno grünblichite qrbeit auf bem Ge.

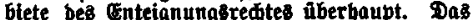
Bert ift nidit bIok fâr bie preubilide \$raxis, fonbern aum far bet Bebrauh in allberen Bunbesftaaten unb flix bie Theorie bon aufser. orbentlifiem nubent. . Go fteht bas gBert in jeber binflat auf ber \$ahe ber æBiffenfhaft."

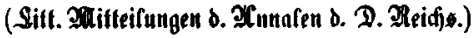

\section{Verlag von VEIT \& COMP. in Leipzaipzig.}

Soeben erschien :

\section{Die Trennungsbefugnis des Civilrichters}

nach $\S 145$ der Civilprozessordnung.

$$
\text { Von }
$$

Dr. Gerhard Hübler.

gr. 8. 1902. geh. $3 \%$.

\section{Der Einredebegriff}

des

Bürgerlichen Gesetzbuch es in seiner praktischen Bedeutung von

Dr. Ernst Suppes.

gr. 8. 1902. geh. 1 A 50 F. 



\title{
Entichetoungen
}

\author{
bes \\ $\mathfrak{A} \mathfrak{e} \mathfrak{i} \mathfrak{d} \mathfrak{g} \mathfrak{g} \mathfrak{e} \mathfrak{x} \mathfrak{i} \mathfrak{d} \mathfrak{t} \mathscr{g}$ \\ ఫerausgegeben \\ bon \\ Den Alitgliedera des (beridtghofes \\ und Der Reid)ganwalt/daft.
}

Entifheibungen in (Eivilladyen.

\author{
Neute Folge. \\ Zweiter gaand.
}

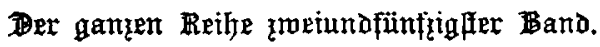

exipzig,

Berlag von Beit \& Comp.

1903 


\title{
(Entjdeidungen
}

\author{
Deaె \\ $\mathfrak{A} \mathfrak{e} \mathfrak{i} \mathfrak{d} \mathfrak{g} \mathfrak{g} \mathfrak{e} \mathfrak{x} \mathfrak{i} \mathfrak{d} \mathfrak{t} \mathfrak{G}$ \\ in \\ Siviliaden.
}

Reute Folge.

Bweiter gando.

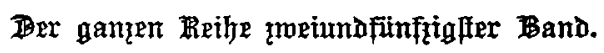

Leipzig,

Berlag yon Beit \& Emp.

1903 


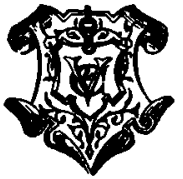

Drud oon Deegger \& \$Bittig in Seipzig. 